\title{
5 Wie kann ich mich weiter informieren?
}

\subsection{Information über das Internet und Printmedien}

Als erste Anlaufstelle ist die Internetrecherche sicherlich nicht geeignet, denn hier droht schnell Desinformation. Zur Vertiefung des Arztgespräches und der therapeutischen Optionen kann das Internet jedoch hilfreich sein. Mittlerweile bieten spezialisierte Kliniken und Behandlungszentren einen guten Überblick über das Behandlungsrepertoire. Auch viele Implantatehersteller bieten interessierten Patienten weiterführende Informationen zu ihren Produkten. Insgesamt ist es erfreulich, dass der Informationsfluss sehr viel transparenter geworden ist.

Printmedien liegen hin und wieder in Arztpraxen oder den Wartebereichen der Kliniken aus und haben oft pseudoredaktionellen Charakter. Häufig wird ein bestimmter Arzt (nämlich der, der die Anzeige bezahlt hat) und/oder eine Prozedur beworben, ohne einen kritischen Überblick zu gewährleisten.

Ist allerdings der Behandlungspfad besprochen und festgelegt, können OP-Beschreibungen, z. B. der Implantatehersteller, sehr hilfreich sein.

\subsection{Empfehlung durch Freunde und Bekannte}

Dieser Weg ist sicherlich der am häufigsten eingeschlagene. Man fragt sich im persönlichen Umfeld durch und wird sicherlich rasch jemanden finden, der ähnliche medizinische Probleme hatte oder einen Arzt kennt, der hierbei bereits hilfreich tätig war.

Natürlich kann ein Laie die Behandlungsqualität nicht umfassend beurteilen, aber ein zufriedener Patient darf durchaus seinen Arzt des Vertrauens empfehlen. Persönliche Erfahrungen mit einer medizinischen Therapie sind nicht zu unterschätzen. 